\title{
Enterocutaneous fistulas and Crohn's disease: Clinical characteristics and response to treatment
}

\author{
Silvia Gómez-Senent ${ }^{1}$, Manuel Barreiro-de-Acosta ${ }^{2}$ and Valle García-Sánchez ${ }^{3}$ \\ Departments of Gastroenterology. ${ }^{1}$ Hospital Universitario La Paz. Madrid, Spain. ${ }^{2}$ Hospital Clínico. Santiago de \\ Compostela, A Coruña. Spain. ${ }^{3}$ Hospital Reina Sofía. Córdoba, Spain
}

\begin{abstract}
Introduction: the low prevalence of enterocutaneous fistulas (ECF) in patients with Crohn's disease (CD) justifies the scarcity of studies that analyze the effect of medical treatment and the need for surgery in these patients. The objective of this study was to evaluate the clinical characteristics of patients with $\mathrm{CD}$ who have ECF and the response to different treatments.

Materials and methods: patients with $\mathrm{CD}$ who presented ECF fistula between 1970 and 2009 in three tertiary centers in Spain were analyzed retrospectively for their clinical characteristics, response to treatment as well as the correlation between the response to biological therapies for ECF and perianal disease. The inflammatory activity of the disease was measured using the Harvey-Bradshaw index. The definitions of partial or complete response were based on the treating physician's judgment.

Results: twenty six patients were included (18 women); in more than half of cases (67\%), anti-TNF treatment improved fistula drainage. Postoperative fistulas responded better to anti-TNF treatment than did spontaneous fistulas (80 versus $57 \%$ ). One hundred percent and $60 \%$ of spontaneous and postoperative fistulas closed after surgery, respectively.

Conclusions: although the majority of patients required surgery as the ultimate treatment, anti-TNF drugs improved fistula output in an acceptable percentage of patients who were treated. Therefore, this may be a treatment strategy prior to surgery in a select group of patients.
\end{abstract}

Key words: Crohn's disease. Enterocutaneous fistula. Treatment.

Gómez-Senent S, Barreiro-de-Acosta M, García-Sánchez V. Enterocutaneous fistulas and Crohn's disease: Clinical characteristics and response to treatment. Rev Esp Enferm Dig 2013; 105:3-6.

Received: 26-04-2012

Accepted: 28-01-2013

Correspondence: Silvia Gómez Senent. Department of Gasatroenterology. Hospital Universitario La Paz. Paseo de la Castellana, 261. 28046 Madrid, Spain e-mail: silviagsenent@gmail.com

\section{INTRODUCTION}

Fistulas are a very common and significant complication in Crohn's disease (CD). They are themselves defined as a clinical pattern of the disease. They originate through transmural extension of the inflammatory process between two adjacent organs. They are seen in between 13 and $48 \%$ of patients, with a cumulative risk of 33 and $50 \%$ at 10 and 20 years after diagnosis, respectively. They are classified as internal when they communicate between two viscera and external when they originate in the ileum or colon and terminate in the skin (1).

Spontaneous enterocutaneous fistulas (ECF) originate in the ileum and are open to the skin of the abdominal wall. They are rare, $0.3 \%$. They usually have a high output, hypoalbuminemia and malnutrition. They rarely respond to medical treatment and ultimately require surgical treatment (2). There is little experience with biological drugs in these types of fistulas, but their use is reasonable, especially in patients with moderate-severe disease activity. Conversely, postoperative fistulas appear during the postoperative period of a patient with abdominal surgery. They originate in the ileum or colon and drain in the skin through the ostomy scar. The majority of these close spontaneously or with skin care, antibiotics and fluids.

The majority of studies that analyze the prevalence or treatment (observational or randomized) of fistula disease have been done on patients with perianal fistulas, which is why if there is a dearth of controlled studies on non-perianal fistula disease $(3,4)$. Only subanalysis of multi-site studies allows the effect of pharmacological treatment versus placebo to be analyzed in patients with enteroenteric and enterocutaneous fistulas.

The objective of this study was to evaluate the clinical characteristics of patients with $\mathrm{CD}$ who have ECF and the response to different treatments, with special attention to biological therapies. 


\section{MATERIALS AND METHODS}

\section{Patient selection}

All patients with a documented history of CD who developed an ECF during their clinical progress from 1970 to 2009 were included retrospectively. All patients were recruited in the following tertiary hospital centers: Hospital Universitario La Paz, Madrid, Hospital Reina Sofía, Córdoba, and Hospital Clínico, Santiago de Compostela.

Regarding the patients' clinical characteristics, epidemiological factors such as year of diagnosis, presence of a family history of inflammatory bowel disease, tobacco use, and the presence of extraintestinal manifestations were recorded. In addition, the disease phenotype was determined using the Montreal Classification and the level of disease activity was based on the Harvey-Bradshaw index.

ECF was defined as postoperative or spontaneous depending on whether or not they were secondary to bowel surgery for the underlying disease. Based on location, they can be divided into: enterocutaneous, if the fistula developed between the small intestine and the skin, and colocutaneous if the fistula developed between the colon and the skin. Diagnosis was made after physical examination and a computed tomography (CT) or magnetic resonance (MRI) study. Clinical signs of ECF were described as the passage of gas or feces through an external orifice as well as the presence of abdominal pain.

\section{Treatment}

The following treatments were considered: antibiotics, thiopurines, infliximab, adalimumab and surgery. The antibiotics used were metronidazole and/or ciprofloxacin; azathioprine was the immunosuppressant used in all patients who received this treatment. Patients who underwent surgery had resection of the bowel segment that gave rise to the fistula as well as resection of the fistula tract to the skin. The indication for surgery, the response or lack thereof to treatment of both the disease and the ECF, as well as the correlation between one and the other was evaluated in all patients. The definitions of partial or complete response were based on the judgment of the treatment physician, with a retrospective measurement made using the HarveyBradshaw index.

\section{Statistical methods}

First, a descriptive analysis of the sample was made, calculating the mean \pm SD for the quantitative variables and the percentage for qualitative variables. Analysis of the level of association was made using Fisher's exact test, the Kappa index and the Wilcoxon test.

\section{RESULTS}

Twenty six patients ( 8 men/18 women) with ECF were included with a mean age of 45.7 years. Fifty four percent were smokers. Based on the Montreal classification, $88 \%$ were $\mathrm{A} 2,81 \% \mathrm{~B} 3$ and $65 \% \mathrm{~L} 3$, with perianal involvement in $58 \%$ of patients. Table I shows the patients' clinical and demographic characteristics. ECF was spontaneous in $57.7 \%$ and occurred after surgery in $42.3 \%$, the most common location being enterocutaneous (small intestine) $(88.5 \%)$. The mean time until onset of ECF from the time of CD diagnosis was 107 months.

Moderated disease activity was registered in $46.7 \%$ of patients with spontaneous fistulas while $45.5 \%$ with postoperative fistulas were inactive at diagnosis.

Ninety-six pecent of patients received antibiotics (metronidazole and ciprofloxacin), with non-response rates of 63.6 for postoperative fistulas and $71.4 \%$ for spontaneous fistulas. $92.3 \%$ of patients were treated with thiopurines, with $87.5 \%$ of postoperative fistulas and $92.3 \%$ of spontaneous fistulas not responding to treatment.

A total of 24 patients were treated with anti-TNF. Three of the 24 patients had complete closure of the fistula, with improvement in $54 \%$ while 8 patients did not have a response. Depending on the type of fistula, anti-TNF treatment led to closure of the fistula in 1 of 14 spontaneous fistulas and 2 of 10 postoperative fistulas. Fistula improvement was found in $7(50 \%)$ and $6(60 \%)$ patients, respectively. There was no response in 6 of the 14 spontaneous fistulas (43\%) and in 2 of the 10 postoperative fistulas. Figure 1 describes the ECF response rates to anti-TNF treatment according to whether they were spontaneous or postoperative.

Regarding treatment, 23 patients received infliximab with a mean treatment duration of 18 months, of which 7 of these were switched to adalimumab due to lack of response. Meanwhile, 1 patient did not receive previous biological treatment and was treated with adalimumab for a mean duration of 16 months. Infliximab led to complete closure in 1 of 9 patients with spontaneous fistula and 2 of

Table I. Patients' clinical characteristics

\begin{tabular}{ll}
\hline Gender & 8 men, 18 women \\
\hline Smokers & $54 \%$ \\
\hline Age & 45.7 years \\
\hline Montreal Classification & \\
A1, A2, A3 & $3.8 \%, 88.5 \%, 7.7 \%$ \\
B1, B2, B3 & $0 \%, 19 \%, 100 \%$ \\
L1, L2, L3, L4 & $19 \%, 11.5 \%, 65.4 \%, 3.8 \%$ \\
$p$ & $58 \%$ \\
\hline Spontaneous fistula & $57.7 \%$ \\
Postoperative fistula & $42.3 \%$ \\
\hline
\end{tabular}


7 patients with postoperative fistulas. Improvement or partial closure was observed in 3 of the spontaneous fistulas (33\%) and in 3 of the postoperative fistulas (43\%). There was no response in 5 and 2 cases, respectively. Closure was not achieved for either the spontaneous or postoperative fistulas. However, improvement was seen in 4 of the 5 patients with spontaneous fistulas $(80 \%)$ and in 3 of the 3 patients with postoperative fistulas (100\%). Eleven patients (5 postoperative and 6 spontaneous fistulas) required surgery, with complete closure rates of 60 and 100\%, respectively (Figure 2). Of the patients with perianal involvement, the ECF response to treatment with infliximab or adalimumab correlated with the response to treatment of perianal disease $(\mathrm{k}=0.774$ and 1$)$.

\section{DISCUSSION}

ECF rarely responds to medical treatment and a high percentage of patients ultimately require surgical treatment (1-5). If the fistula is postoperative and occurs within the first 7 days, re-do surgery will be required. Nevertheless, if it appears later but within 60 days, a watchful approach with enteral or parenteral nutrition may be taken given that these usually close if there is no associated obstructive component. One special situation is that of long-term CD patients with multiple enterocutaneous fistulas because their nutritional status, complexity of the fistulas and tolerance to feeding are highly variable. In these cases, the decision to treat medically or surgically should be evaluated on an individual basis according to the factors mentioned above.

In our series, the mean time from the diagnosis of $C D$ to the onset of ECF was 107 months, meaning we have to assume that the type of treatment to be used will depend on the factors mentioned above. Therefore, neither medical nor surgical treatments have shown a high percentage of ECF closure.

It should be noted that although biological drugs appeared in 2000, we started collecting data from 1970 since the majority of patients treated with anti-TNF drugs

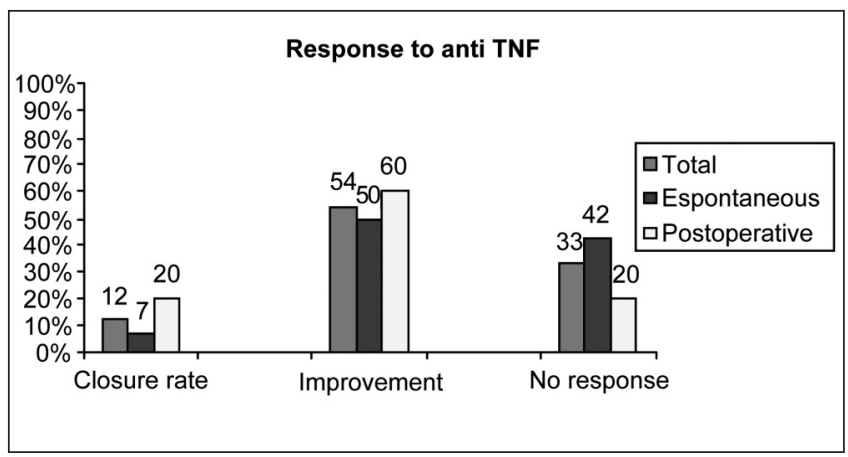

Fig. 1. ECF response to anti-TNF treatment according to spontaneous and postoperative fistulas. in our series had a long disease history. The mean time to the onset of ECF was 107 months from CD diagnosis. Because of this, a large portion of our patients were diagnosed long before the era of biological drugs. They were treated with antibiotics, immunosuppressants and even surgery. Later, with the arrival of these drugs, 24 of the 26 patients in our study started treatment with anti-TNF agents due to the poor response to previous treatments.

There is currently no controlled study that analyzes ECF response to treatment, so we cannot make any comparison with previously-published studies in this section. The data are very limited and consist primarily of small case series. Cougard et al. (6) describe three patients with $\mathrm{CD}$ who developed ECF in the anastomosis after undergoing bowel resection for a stenosing disease pattern. Ileocolonoscopy revealed disease recurrence at this level so treatment with anti-TNF was started. After the first, second and third dose, patients described symptomatic improvement and had no output from the fistula after the eighth dose. Treatment of the endoscopic and clinical recurrence was probably the key to healing the ECF in these patients.

The low response rate in our study was notable, both for spontaneous and postoperative ECF when treated with antibiotics (71.4 and 63.6\%, respectively) and when treated with thiopurines (92.3 and $87.5 \%$, respectively). However, it is clear that although these are the first line of treatment in clinical guidelines, there is no data published on the subject.

A total of 24 patients treated with anti-TNF were included and in more than half of the cases (67\%), anti-TNF treatment improved fistula drainage. Depending on the type of fistula, postoperative fistulas responded better to anti-TNF treatment than did spontaneous fistulas (80 versus $57 \%$ ). Our study revealed that in patients who had associated perianal disease, ECF response to anti-TNF treatment correlated with the response in perianal disease. However, it is clear that in the few studies that have evaluated or compared infliximab in perianal and enterocutaneous fistulas, the results have always been worse when compared with enterocutaneous fistulas. There has even been a case described of an ECF that resolved with infliximab in a patient who did not have $\mathrm{CD}(8)$. These data may be explained by the

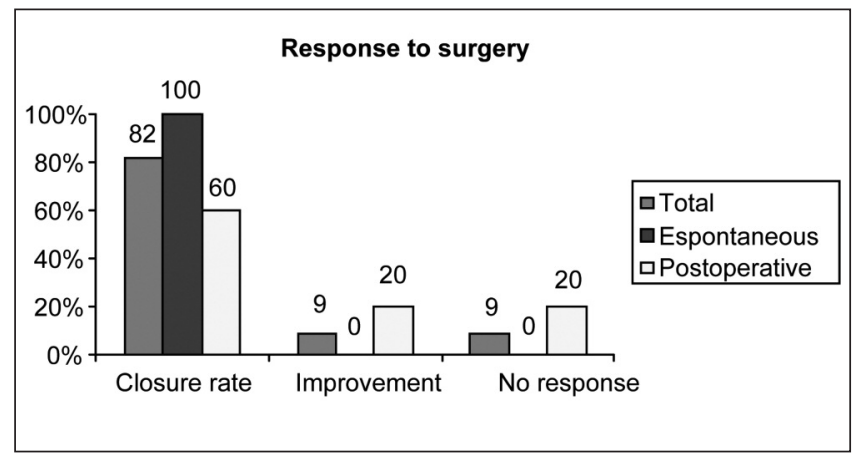

Fig. 2. ECF response to surgical treatment according to spontaneous and postoperative fistulas. 
fact that ECF has a pathophysiology distinct from perianal fistulas. In fact, there is currently a study under way in the United Kingdom that will evaluate the presence or absence of TNF expression on ECF. The results of these studies will help us learn the true efficacy of biological therapies in these patients (9).

Of all the patients who required surgery, 60 and 100\% of postoperative and spontaneous fistulas, respectively, had complete closure. There is a retrospective Canadian study with more than 50 patients in which the authors concluded that surgery was the best option in these patients (10). Fries et al. presented three cases of patients with stenosing CD who, after requiring bowel surgery, developed ECF that was treated with combination anti-TNF agents and surgery with good results (11). A good medical-surgical combination with monitoring of times may be the best treatment option in these patients.

The data obtained in our study were obtained from a retrospective series. Despite the known limitations of this type of study, we can conclude that although the majority of patients required surgery as the definitive treatment, antiTNF drugs improved fistula output in an acceptable percentage of patients. It could therefore be considered as a treatment strategy prior to surgery in a select group of patients. Controlled studies are needed in order to evaluate the efficacy of the different medical and surgical treatments available in patients with ECF.

\section{REFERENCES}

1. Orangio GR. Enterocutaneous fistula: medical and surgical management including patients with Crohn's disease. Clin Colon Rectal Surg 2010;23:169-75.

2. Ulloa Márquez E, Manzano Alonso ML, Martínez Montiel P, SolísHerruzo JA. Fistulizing Crohn's disease. Rev Esp Enferm Dig 2007;99:604-5.

3. Nunes J, Santos PM, Tavares L. Complete resolution of enterocolic fistulas with infliximab. BioDrugs 2010;24:28-30.

4. Kouklakis G, Efremidou EI, Zezos P, Liratzopoulos N, Souftas VD, Gatopoulou A, et al. Adalimumab - an effective and promising treatment for patients with fistulizing Crohn's disease: a case series. J Med Case Reports 2011;5:109.

5. Dignass A, G. Van Assche G, Lindsay JO, Lemann M, Söderholm J, Colombel JF, et al. The second European evidence-based consensus on the diagnosis and management of Crohn's disease: Current management. J Crohns Colitis 2010;4:28-56.

6. Cougard PA, Desjeux A, Berdak S, Ezzedine S, Barthet M, Grimaud JC. Healing of anastomotic enterocutaneous fistulae due to Crohn s disease by anti TNF alpha antibodies. Inflamm Bowel Dis 2010;16:1622-33.

7. Parsi MA, Lashner BA, Achkar JP, Connor JT, Brzezinski A. Type of fistula determines response to infliximab in patients with fistulous Crohn's disease. Am J Gastroenterol 2004;99:445-9.

8. Date RS, Panesar KJ, Neilly P. Infliximab as a therapy for nonCrohn's enterocutaneous fistulae. Int J Colorectal Dis 2004;19:603-6.

9. Rahbour G, Hart AL, Al-Hassi HO, Ullah MR, Gabe SM, Knight SC, et al. Prospective study of immunological factors in non-inflammatory bowel disease enterocutaneous fistulas. BMC Surg 2011;11:12.

10. Poritz LS, Gagliano GA, McLeod RS, MacRae H, Cohen Z. Surgical management of entero and colocutaneous fistulae in Crohn's disease: 17 year's experience. Int J Colorectal Dis 2004;19:481-5.

11. Fries W, La Malfa G, Costantino G, Repici A, Mazziotti S, Navarra G. Combined approach with biologics and surgery for enterocutaneous fistulas in Crohn s disease. Inflamm Bowel Dis 2011;17:671-2. 